\title{
Search for Leptoquarks and Contact Interactions at HERA
}

\author{
Xiang Liu* \\ Address: ZEUS DESY, Notke. str. 85, Hamburg, Germany, 22603 \\ E-mail: 'xliu@mail. desy. de!
}

ABSTRACT: Recent results of the search for leptoquarks (LQ) and contact interactions (CI) at HERA, based on $e^{+} p$ and $e^{-} p$ data collected by ZEUS and H1 from 1994 to 2000, are presented. No evidence was found in either analysis. Therefore, limits for certain LQ and CI models were derived. In some models, HERA has stricter limits than LEP and TEVATRON.

\section{Introduction}

With the $e^{ \pm} p$ data collected at HERA collider, the neutral current (NC) and charge current (CC) deep inelastic scattering (DIS) cross sections can be precisely measured in the high $x$ or high $Q^{2}$ regions. In these regions, possible new physics beyond the standard model (SM), such as LQ and CI, might have visible effect on the cross section. Therefore, the HERA data might be sensitive to the LQ and CI search in these regions. Furthermore, by combining $e^{+} p$ and $e^{-} p$ data, HERA has a unique opportunity to discover certain LQ and CI models which are sensitive to different initial lepton flavors.

From 1994 to 1997, both ZEUS and H1 collected approximately $48 \mathrm{pb}^{-1}$ of $e^{+} p$ data, and

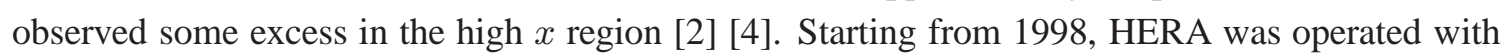
the proton beam energy increased from $820 \mathrm{GeV}$ to $920 \mathrm{GeV}$. And the center of mass energy was increased from $300 \mathrm{GeV}$ to $318 \mathrm{GeV}$. By year 2000, ZEUS and $\mathrm{H} 1$ collaborations had each collected approximately $110 \mathrm{pb}^{-1}$ of $e^{+} p$ and $15 \mathrm{pb}^{-1}$ of $e^{-} p$ data. Therefore, It is interesting and exciting to update the previous LQ and CI search results with higher energy and more luminosity.

\section{Search for Leptoquarks}

Generally speaking, the leptoquark is a high-mass resonant state carrying both baryon and lepton numbers $(\mathrm{F}=\mathrm{L}+3 \mathrm{~B}$, where $\mathrm{F}$ is the LQ's fermion number). It is predicted by theories including

\footnotetext{
${ }^{*}$ Speaker.
} 


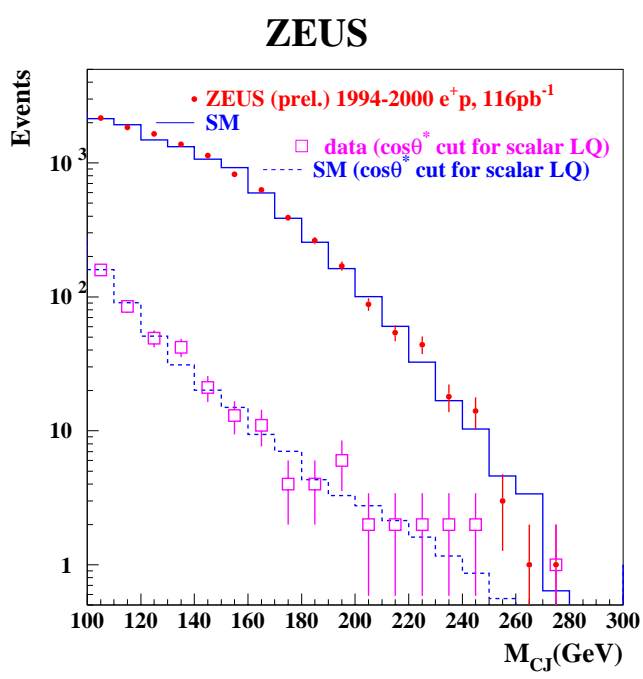

Figure 1: Comparison of the observed events (dots) and SM expectations (histogram) for the reconstructed $e^{+}$-jet invariant mass. Also shown are events after $y$ or $\cos \theta^{*}$ cut, which is optimized for scalar LQ searching.

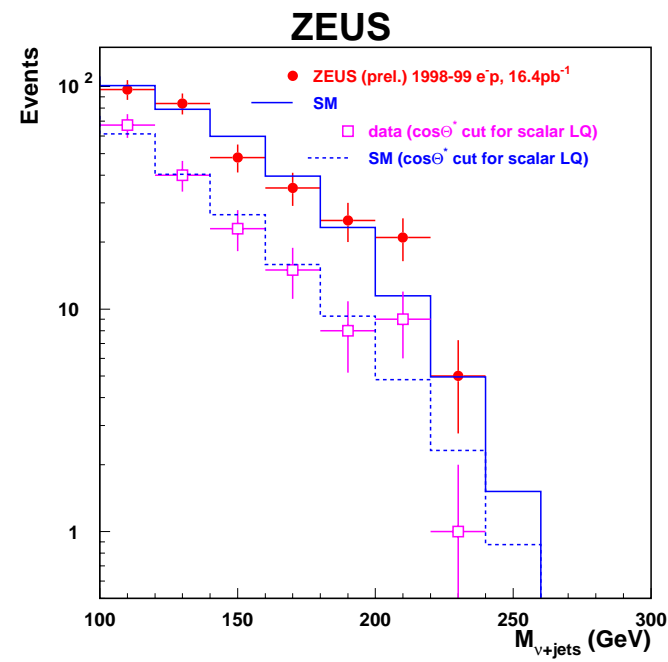

Figure 2: Comparison of the observed events (dots) and SM expectations (histogram) for the reconstructed $\nu$-jet invariant mass. Also shown are events after $y$ or $\cos \theta^{*}$ cut, which is optimized for scalar LQ searching.

BRW leptoquarks [i], proposed by W. Buchmüller, R. Rückl and D. Wyler, and squarks in Rparity violating supersymmetry. At HERA, LQ may be produced directly from s-channel $e^{ \pm}$ quark fusion, and decays into $e^{ \pm}$-quark, or $\nu(\bar{\nu})$-quark, which would yield a peak in the lepton-jet invariant mass spectrum for the $e^{ \pm} p \rightarrow e^{ \pm}(\nu) X$ reactions. LQ may also be exchanged through u-channel, affecting the high $Q^{2} \mathrm{SM}$ cross section without giving an obvious resonance. Therefore, both ZEUS and H1 searched general leptoquarks through two channels: NC DIS and CC DIS. To select CC-like LQ candidate events, it is required that the net transverse momentum of the event be large, due to the undetected neutrino. To select NC-like LQ candidates, a high energy electron is required in the final state.

By studying the $e^{+}$-jet invariant mass $\left(M_{e j}\right)$ spectrum with HERA $48 \mathrm{pb}-1 e^{+} p$ data taken

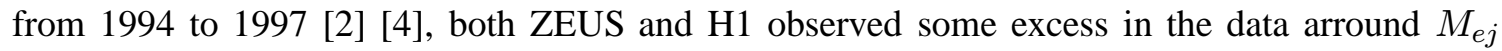
200-210 GeV. Both ZEUS and H1 have updated their results by adding the latest $68 \mathrm{pb}^{-1} e^{+} p$ data taken from 1999 to 2000. Both collabrations have found no more significant excess in the data. The $M_{e j}$ spectrum derived by ZEUS is shown in Fig. 1. The $e^{-}$-jet invariant mass spetrum has also been studied by ZEUS and H1. There is no sign of any deviation from the SM expectation, either.

H1 has also studied the $\nu(\bar{\nu})$-jet invariant mass spectrum with all the $e^{+} p$ and $e^{-} p$ data. ZEUS

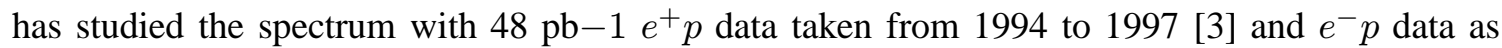
shown in Fig. 2. Again there is no sign of any deviation.

Since HERA has not observed any excess, the limit is set on the LQ mass. When the coupling $\lambda$ is small, the production cross section of a single LQ state can be expressed, under the narrow 
SCALAR LEPTOQUARK $\mathrm{e}^{-} \mathrm{u} \rightarrow \mathrm{LQ} \rightarrow \mathrm{e}^{-} \mathbf{X}, v \mathbf{X}$

Do Run I - H1 Preliminary $e^{-} p$

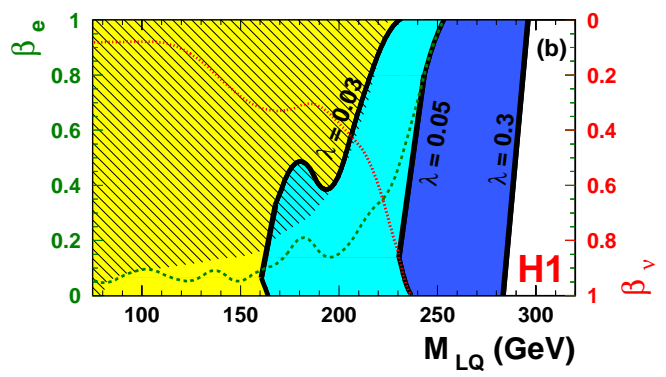

Figure 3: The branching ratios into $e^{-} q$ and $\nu q$ (shown on the left and right axes, respectively) vs. mass for the scalar resonant states with fixed coupling $\lambda$. For each limit curve, the area to the left of the curve is the excluded region.

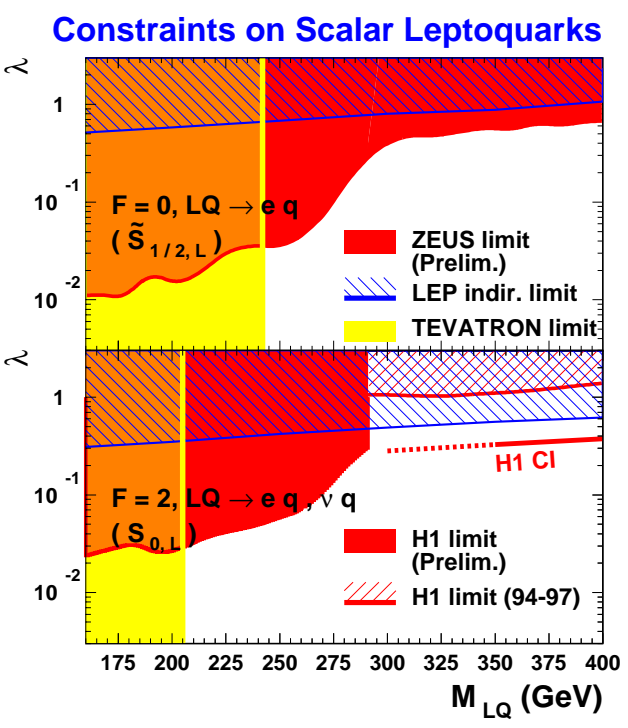

Figure 4: The coupling $\lambda$ limit of two kinds of BRW LQ as function of LQ mass. The HERA results are compared with $\lambda$ limit from LEP and TEVETRON. At TEVETRON, the LQ is assumed to decay into $e^{ \pm} q$ with $100 \%$ branching ratio.

width approximation (NWA), as function of $\lambda$,

$$
\sigma^{N W A}=(J+1) \frac{\pi}{4 s} \lambda^{2} q\left(x_{0}, M^{2}\right),
$$

where $\mathrm{J}$ represents the resonance spin, $q\left(x_{0}, M^{2}\right)$ the quark density evaluated at $x_{0}=M^{2} / s$ and at a virtuality scale of $M^{2}$. Due to the valence and sea quark density difference inside the proton, only $e^{-} p$ data is used for setting the $\mathrm{F}=2 \mathrm{LQ}$ mass limit. The mass limit at $95 \%$ C.L. is set as a function of the LQ decaying branching ratio under different coupling strength, and the results derived by $\mathrm{H} 1$ are shown in Fig. 3. ZEUS has similar results. As shown in the figure, with both $e q$ and $\nu q$ channels, at constant coupling $\lambda$, the LQ mass limit becomes almost independent of the LQ decaying branching ratio. At coupling $\lambda=0.31$, the LQ with mass up to $290 \mathrm{GeV}$ is excluded.

The BRW LQ model assumes $\mathrm{SU}(3) \times \mathrm{SU}(2) \times \mathrm{U}(1)$ invariance, and predicts 14 kinds of $\mathrm{LQs}$, 7 with $\mathrm{F}=0$ and $7 \mathrm{~F}=2$. Some LQs have both $e q$ and $\nu q$ decaying channels with fixed branching ratio $50 \%$. Instead of NWA, here we use full BRW LQ cross section, including the LQ interference term with the SM. Thus we are able to study and set limit on the LQ with mass beyond the HERA kinematic limit. Both $\mathrm{H} 1$ and ZEUS set the limit on the coupling $\lambda$ as a function of the LQ mass. The results of two specific kinds of BRW LQ are shown in Fig. 4. HERA has stronger limits on $\mathrm{F}=0 \mathrm{LQ}$ than LEP.

\section{Search for Contact Interactions}

Some possible high-energy scale new physics, like TeV-scale leptoquark, which is beyond the SM, is beyond the HERA kinematic limit, and this can not be produced as the resonant state. However, 
they might have indirect 'tail' effect in the low-energy range. This effect is described by additional four-fermion vector eeqq terms in the SM lagrangian. (The scalar and tensor terms already have strong limits, therefore they are not considered here.)

$$
\begin{aligned}
& L=\sum_{i, j=L, R} \eta_{i j}^{e q}\left(\bar{e}_{i} \gamma^{\mu} e_{i}\right)\left(\bar{q}_{j} \gamma_{\mu} q_{j}\right) \\
& \eta= \pm \frac{g_{C I}^{2}}{\Lambda^{2}}
\end{aligned}
$$

where the sum runs over electron and quark helicities, $\Lambda$ is the CI effective mass scale, and the coupling strength $g_{C I}=\sqrt{4 \pi}$. It is assumed that all up-type and down-type quarks have the same CI coupling. By switching on or off $\eta_{i j}^{e q}$ with different helicities and flavors, different CI models are studied at HERA by searching for possible deviation of NC DIS cross section $\frac{d \sigma}{d Q^{2}}$ at the high $Q^{2}$ region. ZEUS and $\mathrm{H} 1$ have previously published the CI results with part of the HERA data [5్s.] [6] their results with more luminosity. In order to examine how the CI model would affect the cross section $\frac{d \sigma}{d Q^{2}}$, the effect of one CI model $V V$ is shown in Fig. '占r. The dots represent numbers of the observed events divided by numbers of the SM expectation within $Q^{2}$ bins. The observed numbers of events are well described by the SM. The two dotted lines show how the CI model affects the cross section. Since no deviation from the SM expectation is found, the 95\% C.L. limits are set on the CI mass scale $\Lambda$. The results of all the studied models are shown in table 1. The limits approximately range from 1 to $7 \mathrm{TeV}$.

ZEUS and $\mathrm{H} 1$ have also searched for the Large Extra Dimension (LED) and the quark radius effect, which fall into CI scenario at low energy scale. The LED model is aimed to solve the hierarchy problem by introducing extra dimensions, which in a small volume reduces the Planck mass scale of $10^{19} \mathrm{GeV}$ to effectvie Planck scale $M_{S}$ of the order of TeV. The quark radius model assumes the spatial distribution of the quark charge, thus changing the SM cross section. Without any deviation found in the HERA data, ZEUS and $\mathrm{H} 1$ have set limit on the LED effective mass scale $M_{S}>0.8 \mathrm{TeV}$, and quark radius $R_{q}<0.8 \cdot 10^{-16} \mathrm{~cm}$.
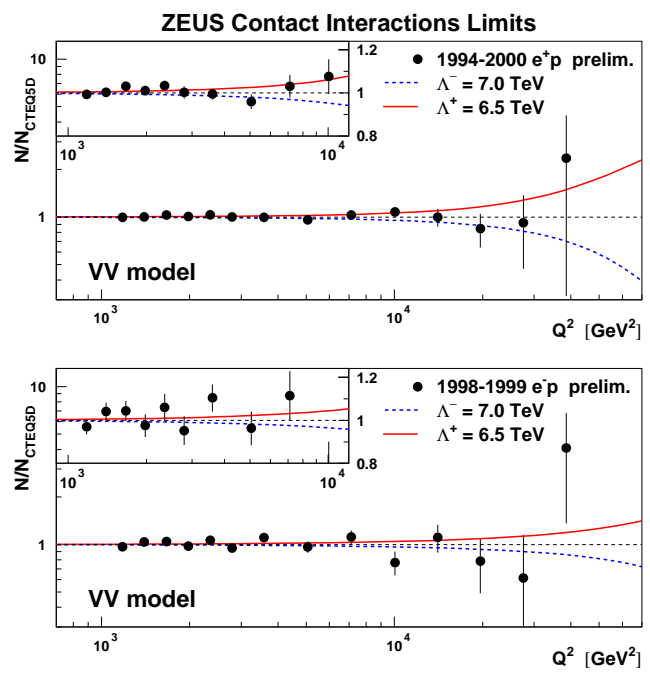

\section{Conclusion}

With the HERA data taken from 1994 to 2000, both ZEUS and H1 have searched for deviation

Figure 5: The effect of CI model VV on NC DIS cross section $\frac{d \sigma}{d Q^{2}}$. The effect becomes visible starting from $Q^{2} 10,000 \mathrm{GeV}^{2}$. of the DIS cross section from the SM prediction, induced by the leptoquark and contact interaction models. Without any significant deviation found, 
95\% C.L. limits are set for the LQ and CI models. For the Yukawa coupling of electromagnetic strength, the LQs with masses up to $290 \mathrm{GeV}$ are excluded. The CI mass scale below 1 to $7 \mathrm{TeV}$, depending on the parameters of the CI models, is also excluded.

HERA has finished it's luminosity upgrade and is expected to take data starting from year 2002, with approximately four times more luminosity, and also with polarized beams. We expect more exciting results in the near future.

\begin{tabular}{|c|c||c|c||c|c|}
\hline \multicolumn{2}{|c||}{ 1994-2000 $e^{ \pm} p$ 95\% C.L. (TeV) } & \multicolumn{2}{|c|}{ ZEUS } & \multicolumn{2}{c|}{ H1 } \\
\hline \hline Model & $\left(\eta_{L L}, \eta_{L R}, \eta_{R L}, \eta_{R R}\right)$ & $\Lambda^{-}$ & $\Lambda^{+}$ & $\Lambda^{-}$ & $\Lambda^{+}$ \\
\hline \hline LL & $(+1,0,0,0)$ & 1.6 & 2.7 & 1.6 & 4.3 \\
LR & $(0,+1,0,0)$ & 2.2 & 3.8 & 1.8 & 5.4 \\
RL & $(0,0,+1,0)$ & 2.4 & 3.7 & 1.9 & 5.4 \\
RR & $(0,0,0,+1)$ & 1.7 & 2.7 & 1.6 & 4.3 \\
\hline VV & $(+1,+1,+1,+1)$ & 6.2 & 5.6 & 9.2 & 3.0 \\
AA & $(+1,-1,-1,+1)$ & 5.1 & 4.4 & 3.5 & 5.8 \\
VA & $(+1,-1,+1,-1)$ & 3.3 & 3.2 & 3.9 & 4.0 \\
X1 & $(+1,-1,0,0)$ & 3.8 & 2.6 & & \\
X2 & $(+1,0,+1,0)$ & 3.8 & 4.1 & & \\
X3 & $(+1,0,0,+1)$ & 3.7 & 3.6 & 5.9 & 2.0 \\
X4 & $(0,+1,+1,0)$ & 5.0 & 5.0 & 7.4 & 2.1 \\
X5 & $(0,+1,0,+1)$ & 4.0 & 4.1 & & \\
X6 & $(0,0,+1,-1)$ & 2.5 & 3.6 & & \\
U1 & $(+1,-1,0,0)^{e u}$ & 4.0 & 3.6 & & \\
U2 & $(+1,0,+1,0)^{e u}$ & 4.9 & 4.3 & & \\
U3 & $(+1,0,0,+1)^{e u}$ & 5.0 & 4.2 & & \\
U4 & $(0,+1,+1,0)^{e u}$ & 5.7 & 5.1 & & \\
U5 & $(0,+1,0,+1)^{e u}$ & 5.1 & 4.5 & & \\
U6 & $(0,0,+1,-1)^{e u}$ & 2.7 & 3.6 & & \\
\hline
\end{tabular}

Table 1: Couplings $\left(\eta_{L L}, \eta_{L R}, \eta_{R L}, \eta_{R R}\right)$ of different CI models and the $95 \%$ C.L. limits on the effective mass scale $\Lambda$. Each row of the table represents two scenarios corresponding to $\eta>0\left(\Lambda^{+}\right)$and $\eta<0\left(\Lambda^{-}\right)$. The lepton couples to d- and u-quarks, except for the models U1 to U6, in which the lepton does not couple to d-quarks.

\section{References}

[1] W. Buchmüller, R. Rückl and D. Wyler, Phys. Lett. B191 (1987) 442.

[2] ZEUS Collaboration, J. Breitweg et al., Eur. Phys. J. C16 (2000) 253.

[3] ZEUS Collaboration, J. Breitweg et al., Phys. Rev. D63 (2001) 052002.

[4] H1 Collab., C. Adloff et al., Eur. Phys. J. C11 (1999) 447.

[5] ZEUS Collaboration, J. Breitweg et al., Eur. Phys. J. C14 (2000) 239.

[6] H1 Collab., C. Adloff et al., Phys. Lett. B479 (2000) 358. 